\title{
TRANSCRIPTIONAL DYNAMICS OF EARLY DEVELOPING TOMATO (SOLANUM LYCOPERSICUM L.) FRUIT BASED ON RNA-SEQ ANALYSIS
}

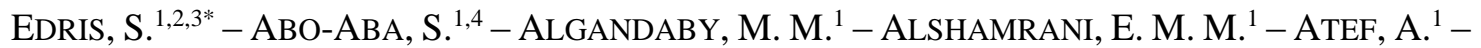 \\ RAMADAN, A. M. ${ }^{1,5}$ - GADALlA, N. O. ${ }^{1,6}$ - SABIR, M. J. S. ${ }^{7}$ - El-DOMYATI, F. M..$^{1,3}$ - MAKKI, R. \\ M. ${ }^{1}$ - HAJRAH, N. H. ${ }^{1}$ - SABIR, M. J. ${ }^{1}$ - ABDEL-HAMID, A. M. E. ${ }^{8}$ - QARI, S. H. ${ }^{9}$ - JANSEN, R. \\ K. ${ }^{1,10}-$ AL-QUWAIE, D. A. H. ${ }^{11}-$ BAHIELDIN, A. ${ }^{1,3}$ \\ ${ }^{1}$ Department of Biological Sciences, Faculty of Science, King Abdulaziz University (KAU), \\ Jeddah, Saudi Arabia \\ ${ }^{2}$ Princess Al-Jawhara Al-Brahim Centre of Excellence in Research of Hereditary Disorders \\ (PACER-HD), Faculty of Medicine, King Abdulaziz University (KAU), Jeddah, Saudi Arabia \\ ${ }^{3}$ Department of Genetics, Faculty of Agriculture, Ain Shams University, Cairo, Egypt \\ ${ }^{4}$ Department of Microbial Genetics, Genetic Engineering and Biotechnology Division, National \\ Research Centre, Giza, Egypt
}

${ }^{5}$ Agricultural Genetic Engineering Research Institute (AGERI), Agriculture Research Center (ARC), Giza, Egypt

${ }^{6}$ Department of Genetics and Cytology, Genetic Engineering and Biotechnology Division, National Research Center, Dokki, Egypt

${ }^{7}$ Department of Computer Science, Faculty of Computer and Information Technology, King Abdulaziz University (KAU), Jeddah 21589, Saudi Arabia

${ }^{8}$ Department of Biological and Geological Sciences, Faculty of Education, Ain Shams University, Cairo, Egypt

${ }^{9}$ Department of Biology, Aljumum University, College, Umm Al-Qura University, Makkah, Saudi Arabia

${ }^{10}$ Department of Integrative Biology, University of Texas at Austin, Austin, TX, USA

${ }^{11}$ Department of Biological Sciences, Rabigh College of Science and Arts, King Abdulaziz University (KAU), Rabigh, Saudi Arabia

*Corresponding author e-mail: seedris@kau.edu.sa; phone: +966-59-366-2384; ORCID ID: 0000-0002-2810-3736

(Received 13 ${ }^{\text {th }}$ May 2019; accepted 20 ${ }^{\text {th }}$ Aug 2019)

\begin{abstract}
Tomato (Solanum lycopersicum L.) is one of the most popular vegetable species grown worldwide. Functional genomic analysis of genes in the early flower and fruit stages are the key factors for genetic enhancement of tomato fruits for increased yield and quality. In the current study, RNA-Seq datasets were generated for the Chico cultivar at six growth stages, 0, 3, 5, 7, 9 and 12 days after pollination (DAP). The computational analysis of transcriptomes and heat map showed close relationships among transcripts regulated at 3 and 5 DAP and at 7 and 9 DAP, whereas transcripts regulated at 12 DAP were more distant. Annotated transcripts and KEGG analysis showed that starch and sucrose pathway experienced the most dynamic changes resulting in the synthesis of several soluble sugars, e.g., sucrose, $\beta$-D-fructose, $\alpha$-D-glucose, trehalose, maltose and 1,4- $\beta$-D-xylan. Enzymes
\end{abstract}


activated in this pathway included levansucrase, phosphotransferse, invertase, pectin methyl esterase (PME), glucan synthase and cellulase. In general, the results provided improved insights into the mechanisms underlying early fruit development in tomatoes.

Keywords: Chico III, analysis, pPCR, KEGG, starch and sucrose pathway, PME2.1, WIV-1(sacA), PTS (scrA), LS (sacB), cellulose (phd) and sphl(cre or glucan synthase)

\section{Introduction}

Tomato (Solanum lycopersicum L.) is one of the most important vegetable crops worldwide due to its economic and agronomic importance (Zhang et al., 2016). Cultivated tomatoes are diploid with 12 chromosomes and a genome size of approximately $950 \mathrm{Mb}$ with 35,000 genes (Guigó Serra et al., 2012) and wild forms range from diploids to hexaploids (Bonierbale et al., 1988; Gupta et al., 2013). Tomato fruit development begins with fertilization and ends at the red ripe stages (Gillaspy et al., 1993; Picken, 1984). Wild tomatoes are important for tomato breeding and also cultivating plants belonging to family Solanaceae because information gained from studies in tomato genome sequencing can be applied to these plants (Kimura and Sinha, 2008).

Functional genomic analysis at the fruit ripening stage with mature, full-sized green fruit has been studied intensively (Gapper et al., 2014), while studies at earlier stages of fruit development are limited. The early developmental stage is very important for tomato fruit formation because the fruit weight and size are widely dependent on the pericarp cell number which is genetically controlled during cell division (Xiao et al., 2008), which leads to cell expansion and increases in average cell size. Transcription factors organizing early steps of the development also appear to have roles in tomato flower and fruit development (Silva et al., 2014). DNA replication is essential during both phases, although it is followed by mitosis in the earlier phase and endomitosis (replication progresses several times without mitosis) in the later phase. The resulting cells can possess multiple genome copies (polyploidy); an important process during cell expansion (Bergervoet et al., 1996).

Parallel with these processes, differentiation of the tissues into pericarp (fruit wall), peel, placenta and locular tissue ("jelly") is completed (Bertin, 2005; Mintz-Oron et al., 2008). Many of the final fruit quality parameters, such as size, shape, taste (soluble solids), texture (wateriness, mealiness), firmness, proneness to cracking, peelability, and properties during processing (viscosity), are co-determined during early stages by signals of some hormones that are stimulated in pericarp developmental stages. Carbohydrates are the main component in early developed tomato fruits. Metabolism of carbohydrates is regulated by many genes that contribute to cell wall synthesis (Zhang et al., 2016).

Web-based resources for genetic control and development of tomato are available at Tomato Genetics Resource Center (TGRC) (http://tgrc.ucdavis.edu) which contains a collection of wild relatives and miscellaneous genetic stocks of tomato (Kimura and Sinha, 2008). Expressed sequence tags (ESTs) library largely replaced microarray technologies in most plant systems because it provides a large-scale picture of gene expression during ripening in tomato (Gapper et al., 2014) and responses to treatment or environmental stresses (Bahieldin et al., 2015a). However, RNA-Seq provides an accurate statistical assessment of gene expression and clusters of genes based on their expression patterns (Bahieldin et al., 2015b). Therefore, RNA-Seq datasets of gene expression in tomato were generated in the present study and verification of gene 
expression profiles involved in several metabolic pathways shed the light on genetic mechanisms that might regulate early developmental stage and the formation of fruit during ripening stage in tomato.

\section{Materials and methods}

\section{Plant material}

Tomato cv. Chico III seeds were germinated in pots filled with Peat moss soil and grown in the glasshouse under controlled conditions (16-h-light/8-h-dark cycle at $21 \pm 2{ }^{\circ} \mathrm{C}$ (day/night), light intensity of $\sim 200 \mathrm{uE} \mathrm{m}^{-2} \mathrm{~s}^{-1}$ for the 16 -h photoperiod and $80 \%$ humidity) (Zhan et al., 2018). Recovered plantlets were watered every other day for 60 days until buds started to develop. Then, flower and bud samples were taken at six growth stages, i.e., 0 day after pollination (DAP), 3 DAP, 5 DAP, 7 DAP, 9 DAP and 12 DAP. Each sample was collected from two pots with four plants each in order to recover enough RNA for further analysis.

\section{RNA extraction, library construction and sequencing}

Total RNAs were extracted using SV Total RNA Isolation System (Promega) and the Truseq $^{\mathrm{TM}}$ RNA Sample Prep Kit (Illumina) was utilized for library construction following the manufacturer's instructions. Then, the library was shipped to Beijing Genomics Institute (BGI) in China for sequencing on Illumina HiSeq ${ }^{\mathrm{TM}} 2000$. For every sequencing read, the indexes were trimmed using SeqPrep program (https://github.com/jstjohn/SeqPrep) and the paired ends were merged . The remaining optimized reads were mapped to the tomato reference genome (ftp://ftp.solgenomics.net/tomato_genome/annotation/ITAG2.3_release/) using Tophat (Trapnell et al., 2009) and SOAPaligner/soap2 software (http://soap.genomics.org.cn) (Robinson et al., 2010). The mapped reads were then assembled with Cufflink (Trapnell et al., 2010). The experiment was submitted to National Center for Biotechnology Information (NCBI) as BioProject accession number PRJNA298353 and as BioSample accession numbers SAMN04214820 to SAMN04214831. RNA-Seq reads were placed in Sequence Read Archive (SRA) database (http://www.ncbi.nlm.nih.gov/Traces/sra/) under the accession number SUB1151548. The differential expression profiling and further annotation analysis utilized the merged and assembled sequences, as well as the original genome annotations.

The Bowtie aligner software of trinity package (http://trinityrnaseq.sourceforge.net/analysis/extract_proteins_from_trinity_transcripts.h tml) was used to predict the mapping of the assembled transcripts reads to the reference genome and annotated genes or open reading frames (ORFs). Sequence-similarity Blast searches of transcripts were conducted against the tomato reference genome, the NCBI genome database (http://www.ncbi.nlm.nih.gov/), the gene ontology (GO) database (http://www.geneontology. org/) and the Kyoto encyclopedia of genes and genomes (KEGG) database (http://www.genome.jp/kegg/) (Kanehisa and Goto, 2000).

GO terms for tomato transcripts were obtained using Blast2GO (v.2.3.5) (http://www.blast2go.org/) with default parameters (Kanehisa and Goto, 2000). Differential expression analysis was performed via Tophat (http://tophat.cbcb.umd.edu/) and Cufflinks (http://cufflinks.cbcb.umd.edu/) programs and RPKM (Reads per kilobase of exon model per million mapped fragments) values were detected within $95 \%$ 
confidence limit. Differential expression was calculated according to the count values of each transcript in the library using edge R software (Robinson et al., 2010). False discovery rate (FDR) of $<0.05$ and $\log ^{2}$ fold-change $\left(\log ^{2} F C\right)$ of $\geq 1$ were used as the thresholds for judging significant differences in transcript expression. Transcripts with $\log ^{2} \mathrm{FC}$ of $<0.25$ were assumed to have no change in expression levels.

\section{Data verification via $q R T-P C R$}

Quantitative real-time reverse transcriptase (qRT-PCR) consideration was performed to verify the expression patterns of randomly selected transcripts of RNA-Seq datasets. Aliquots of the originally purified RNA samples were reverse-transcribed using the PrimeScript RT Reagent kit with gDNA Eraser (Takara, Dalian, China) following the manufacturer's protocol. Eight gene-specific qRT-PCR primer pairs (18-20 bp) (Table 1) were designed using primer Premier 5.0 software (Premier Biosoft International, Palo Alto, CA). These genes are $\operatorname{sacB}(L S)$, scrA(PTS), sacA(WIV-1), $P M E$, xyll, cre (Glucan synthase) and phd (Cellulase) encode enzymes at the starch and sucrose metabolic pathway. RNA-Seq data indicated that these eight genes were regulated during fruit development. Of which, the first four genes were downregulated, while the other three genes were upregulated. qRT-PCR was performed using SybrGreenqRT-PCR master mix (Ruian Biotechnologies, Shanghai, China) in an ABI 7500 FAST Real-Time PCR system (Applied Biosystems, Foster City, CA, USA). PCR conditions were $2 \mathrm{~min}$ at $95{ }^{\circ} \mathrm{C}$, followed by 40 cycles of heating at $95{ }^{\circ} \mathrm{C}$ for $10 \mathrm{~s}$ and annealing/extension at $60{ }^{\circ} \mathrm{C}$ for $40 \mathrm{~s}$ and $72{ }^{\circ} \mathrm{C}$ for $2 \mathrm{~min}$. Three replicates were performed, and the amplicons were used for melting curve analysis to check the amplification specificity. The relative expression level of each gene was calculated as $2^{-}$ $(\Delta \Delta \mathrm{Ct})$ and the housekeeping gene glyceraldehyde-3-phosphate dehydrogenase or GAPDH (accession no. U93208) from S. lycopersicum was used to normalize the amount of template cDNA added in each reaction.

Table 1. Primer sequences for selected genes used for validating RNA-Seq datasets

\begin{tabular}{c|c|c|c}
\hline Gene symbol & $\begin{array}{c}\text { Accession } \\
\text { number }\end{array}$ & Sequence (5'-> 3') & Amplicon (bp) \\
\hline GAPDH & NM_001247874 & $\begin{array}{c}\text { AATTGGCCGATTGGTTGCTC } \\
\text { GAAGGGATCGTTCACTGCGA }\end{array}$ & 73 \\
\hline sacB $(L S)$ & NM_001247250 & $\begin{array}{c}\text { ATCGCGGAGAAGGAAGCAAA } \\
\text { ACTACCCGGTGGCAATGTAG }\end{array}$ & 117 \\
\hline scrA $($ PTS $)$ & NM_001247449 & $\begin{array}{c}\text { TGGCTAGATTGCCACAACTCG } \\
\text { CCAGCTGTTGGGATTCCTCAT }\end{array}$ & 86 \\
\hline sacA $($ WIV-1) & NM_001246913 & $\begin{array}{c}\text { TCGCGAGTTTAGACAAGGCA } \\
\text { CCAAGACCACCTTGAACCGT }\end{array}$ & 103 \\
\hline PME & NM_001247019 & $\begin{array}{c}\text { TGGCGATAAAGCTGAAACTGA } \\
\text { ATAACCACCACCCCACAACC }\end{array}$ & 111 \\
\hline cre $($ glucan synthase $)$ & NM_001247033 & $\begin{array}{c}\text { TCGCTAGCGGAAAATTGCTT } \\
\text { TTCGTCATCCTTTGACGTGC }\end{array}$ & 111 \\
\hline Phd (cellulase $)$ & NM_001247953 & CCCAGACGAGCGTTCAGATT & 98 \\
\hline
\end{tabular}




\section{Results}

\section{Transcriptomic analysis}

RNA-Seq analysis of flowers and buds harvested from tomato cultivar Chico III was done at early fruit development stages ranging from $0,3,5,7,9$ and 12 DAP. Over $24 \mathrm{~Gb}$ of data were generated from six datasets comprising 274,981,532 paired-end reads of $90 \mathrm{bp}$ in length after trimming. The dataset of each sample had $>44$ million reads, which represented a sufficient read density for the subsequent quantitative analysis of gene expression (Table 2).

The clean sequence reads were aligned to the tomato reference genome database allowing two base mismatches. Alignment and assembly resulted in 27,527 transcripts in the transcriptome. This number represents over $65 \%$ of the genes available in the tomato Unigene database (42,257), and 29.2-43.1\% were differentially expressed with $\geq 2$ fold at different time points compared to the control (0 DAP) (Table 2). The percentage of transcripts of different sizes with 150-200, 200-600, 600-1000, 10001600, 1600-2200, 2200-3000 and > 3000 bp were 8.14, 32.53, 20.28, 20.72, 9.44, 5.41 and $3.48 \%$, respectively (Fig. 1). The latter four size categories more likely recover fragments long enough to likely detect function domains of the transcripts. The total number of transcripts expressed at different time points was 4335 in the control group (0 DAP), while 2588 at 3 DAP, 3529 at 5 DAP, 3549 at 7 DAP, 4054 at 9 DAP and 5,968 at 12 DAP.

The heat map indicated that the differential response at 0 DAP was distinct from the later stages (Fig. 2). Transcripts regulated at 3 and 5 DAP and 7 and 9 DAP clustered together, and transcripts regulated at $12 \mathrm{DAP}$ were more distant compared to these four stages. A Venn diagram was constructed to compare the yield of transcripts shared between the following pairs of developmental stages, e.g., 0/3 DAP, 0/7 DAP and 0/12 DAP (Fig. 3). The number of shared transcripts were 8,054 (A+AB + $\mathrm{AC}+\mathrm{ABC})$, $8,750(B+A B+B C+A B C)$ and $11,875(C+A C+B C+A B C)$, respectively out of the total of 14,182 different transcripts. The highest number of shared transcripts was across the three stages (5307) followed by 0 and 7 DAP $(1,871)$. The highest number of unique transcripts $(3,276)$ in the developmental stage pairs was 0/12 DAP followed by 0/7 DAP (981). These unique transcripts across the three developmental stages indicate the occurrence of differential expression during the flowering stages.

Table 2. Number of reads sequenced and mapped to the tomato reference genome

\begin{tabular}{c|c|c|c|c|c|c}
\hline Time point & 0 DAP & 3 DAP & 5 DAP & 7 DAP & 9 DAP & 12 DAP \\
\hline Clean bases (bp) & $4,149,898,380$ & $4,046,486,040$ & $4,033,137,780$ & $4,079,245,860$ & $4,220,982,000$ & $4,218,587,820$ \\
\hline Clean no. reads & $46,109,982$ & $44,960,956$ & $44,812,642$ & $45,324,954$ & $46,899,800$ & $46,873,198$ \\
\hline \% Aligned reads & 82.02 & $81.92 \%$ & $85.21 \%$ & $82.86 \%$ & $85.23 \%$ & $83.75 \%$ \\
\hline$\%$ Unique matches & 36.58 & $49 \%$ & 38.33 & 35.12 & 34.32 & 33.42 \\
\hline $\begin{array}{c}\text { \% Multi-position } \\
\text { matches }\end{array}$ & 45.44 & $45.43 \%$ & 46.88 & 47.74 & 50.91 & 50.33 \\
\hline$\%$ Unaligned reads & 17.98 & $18.08 \%$ & 14.79 & 17.14 & 14.77 & 16.25 \\
\hline $\begin{array}{c}\text { No. differentially } \\
\text { expression genes } \\
(\geq 2 \text { fold) }\end{array}$ & - & 8.045 & 9.465 & 8.750 & 9.678 & 11875 \\
\hline $\begin{array}{c}\text { \% Differentially } \\
\text { expression genes } \\
(\geq 2 \text { fold) }\end{array}$ & - & 29.23 & 34.38 & 31.79 & 35.16 & 43.1 \\
\hline
\end{tabular}




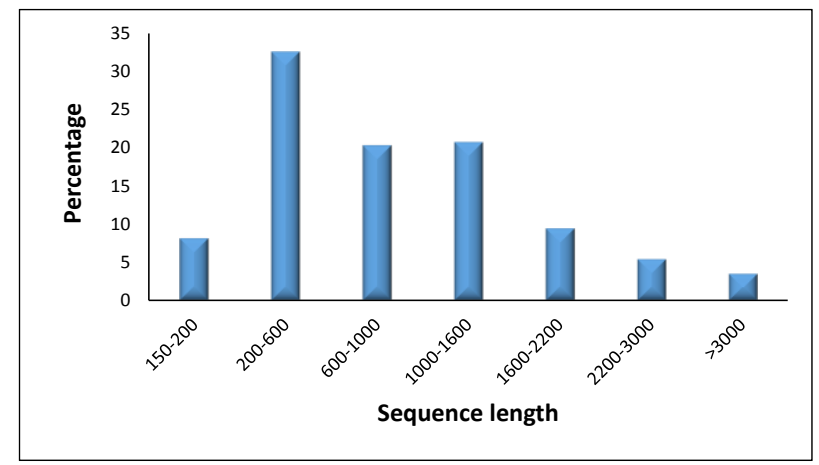

Figure 1. Length distribution of the transcript sequences detected in early fruit development transcriptomes

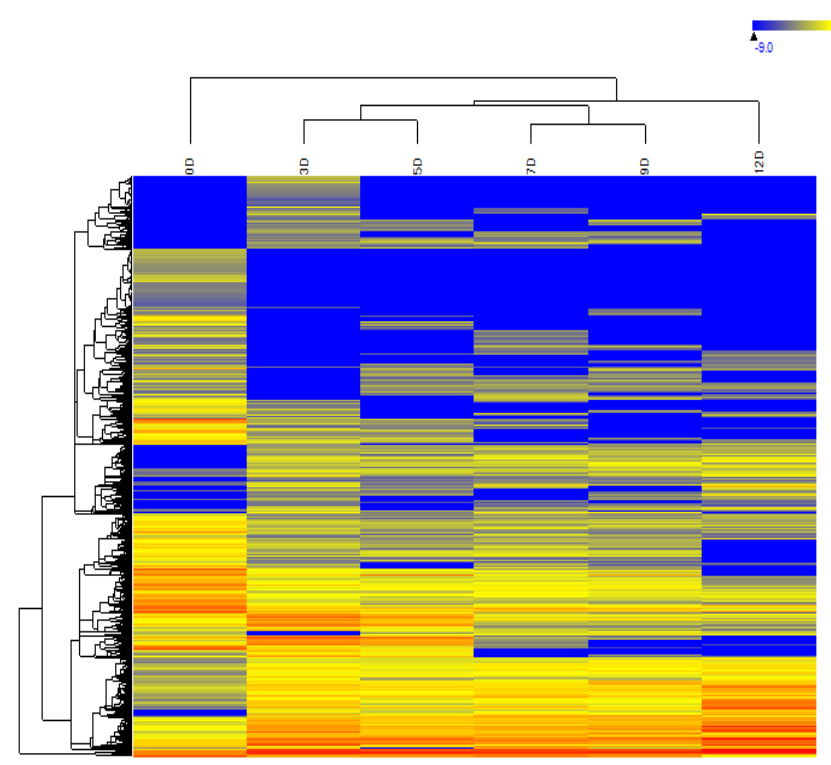

Figure 2. Heat map analysis of all transcripts at the different time courses

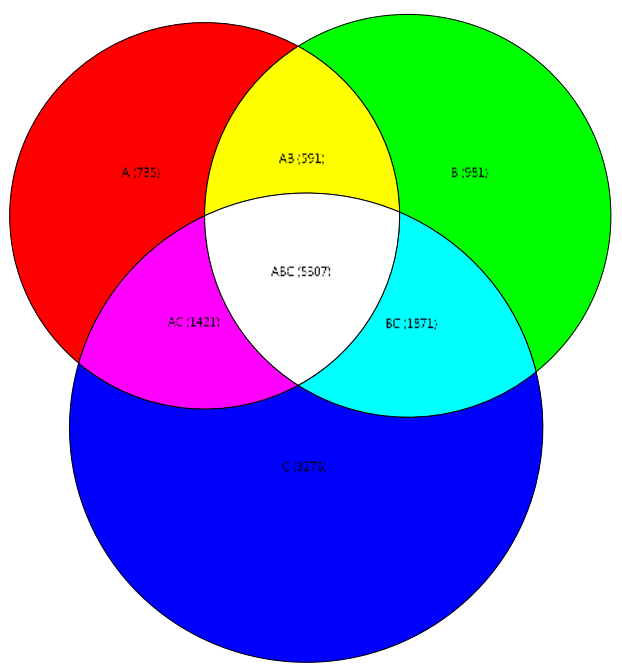

Figure 3. Venn diagram of expressed transcripts at $3(A), 7(B)$ and $12(C)$ DAP compared with those at $O D A P$ 
Annotated transcripts with known function were classified into 52 functional groups, including 13 in cellular component (Fig. 4), 12 in molecular function (Fig. 5) and 19 in biological process (Fig. 6) categories comprising 1,364 regulated transcripts. In the cellular component category, 337 transcripts were regulated. Of which, 96, 75, 56 and 37 transcripts were regulated in the "cell", "organelle", "membrane" and "extracellular region", respectively (Fig. 4). In the molecular function category, 251 transcripts were regulated. Of which, 90, 88, 22 and 13 transcripts were regulated in the "catalytic activity", "binding", transporter activity" and "nucleic and binding transcription factor activity", respectively (Fig. 5). In the biological process category, 778 transcripts were regulated and most of these belong to "metabolic process" (118), followed by "cellular process" (105), "single organism process" (101), "response to stimulus" (68), "biological regulation" (58), cellular component organization or biogenesis (54) and "developmental process" (49) (Fig. 6). Results of the functional groups indicate that the highest activities were at the cell, organelle and membrane levels as well as at the extracellular region level. These activities induced catalysis, binding, transportation and those required for transcription factors binding in the nucleus and cytoplasm. Major processes include metabolism and responses to stimulation and development. The latter two processes are essential for the flower in early developmental stages.

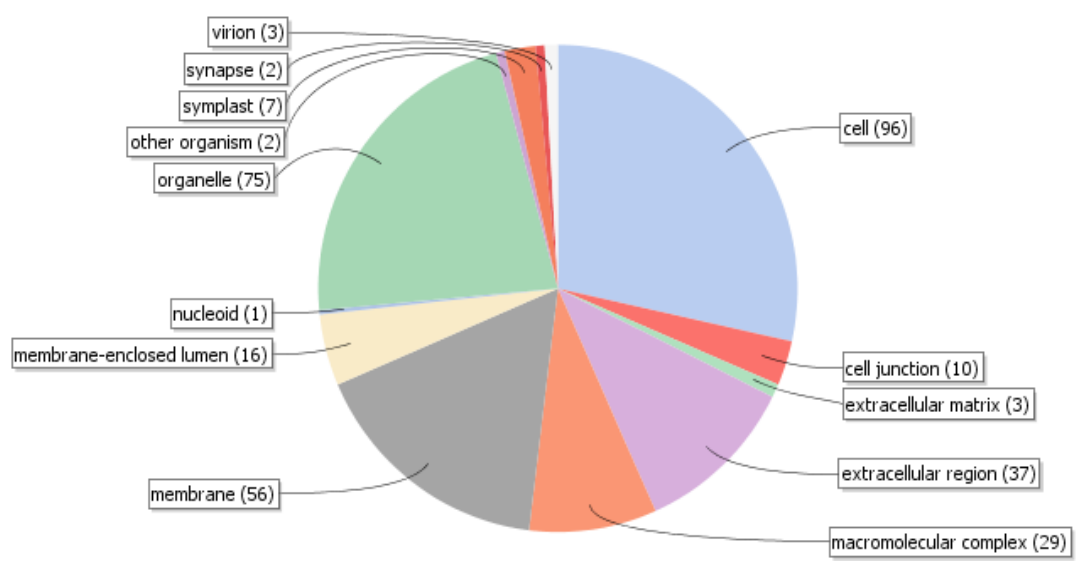

Figure 4. Gene ontology $(G O)$ terms of transcripts by the level 2. All transcripts were assigned to at least one GO term and were grouped under the cellular component category

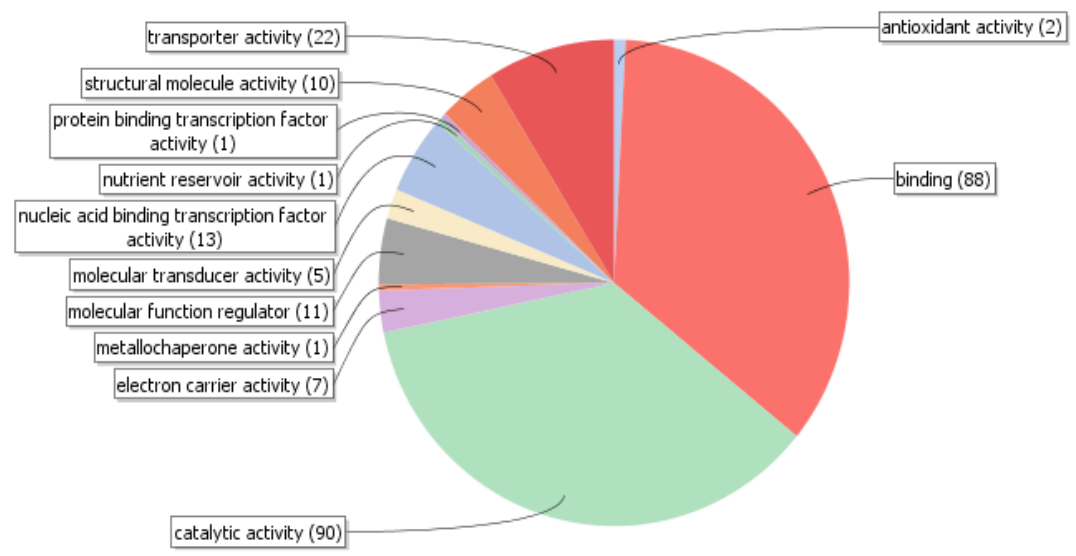

Figure 5. Gene ontology (GO) terms of transcripts by the level 2. All transcripts were assigned to at least one GO term and were grouped under the molecular function category 


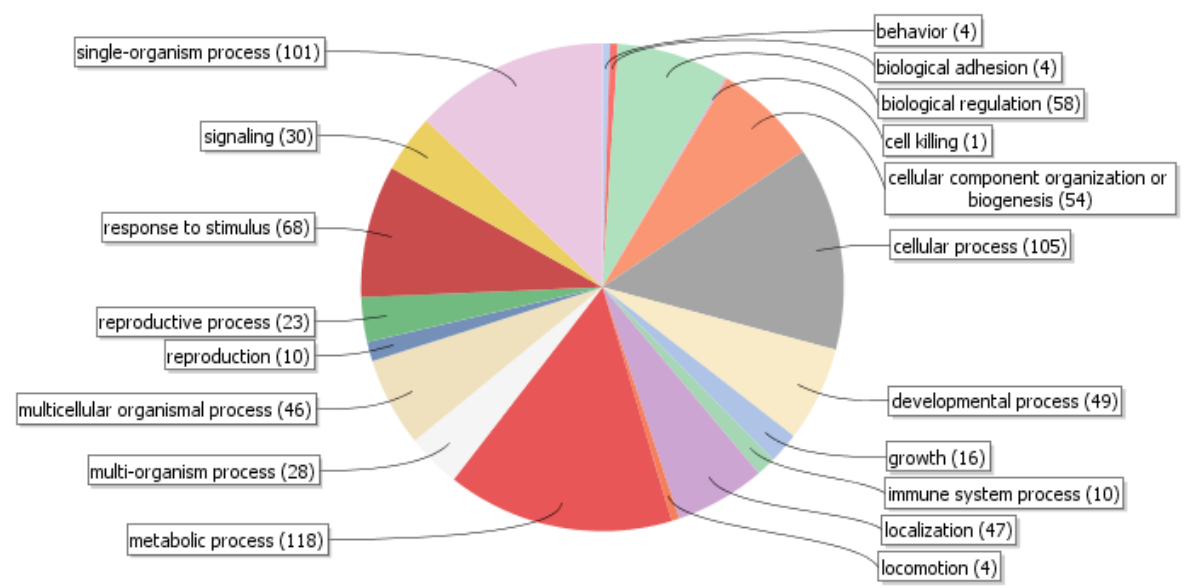

Figure 6. Gene ontology (GO) terms of transcripts by the level 2. All transcripts were assigned to at least one GO term and were grouped under the biological process category and filtered by cutoff $=5$

\section{Verification of RNA-Seq data}

In order to verify the gene expression profiles of enzymes involved in starch and sucrose metabolism obtained from the RNA-Seq datasets, quantitative real-time PCR was utilized. Eight genes were selected for the analysis based on their regulation during starch and sucrose metabolic pathway. They are scrA, sacB, phd, cre, sacA, pg2, pme and xyll (Fig. 7). These genes encode phosphotransferse (EC:2.7.1.69), levansucrase (EC:2.4.1.10), cellulase (EC:3.2.1.4), glucan synthase (EC:2.4.1.34), invertase (EC:3.2.1.26), exopolygalacturonase (EC:3.2.1.67), pectin methylesterase (EC:3.1.1.11) and beta-xylosidase (EC:3.2.1.37).

Figure 8 shows that eight genes were divided into three groups depending on its expression profile, first group were down regulated containing PME2.1, PTS (scrA), Cellulose (phd) and sphl(cre or Glucan synthase), the second pattern was up-regulated $W I V-1(\operatorname{sacA})$ and the third pattern was partially down regulated $L S$ (sacB).

\section{Discussion}

The results are concomitant with those of Silva et al. (2014) who found that miR156targeted $S$. lycopersicum SBP genes were dynamically expressed in developing flowers and ovaries as the cell is active in metabolism and likely to be sensitive to external stimuli. Hydrolase, transferase and oxidoreductase enzymes are predominant across the different stages of fruit development (Fig. 9). Recent reports indicate that one family of the hydrolases, e.g., xyloglucan endotransglycosylase/hydrolase (XTH), plays an important role in the remodeling of cell wall hemicelluloses during ripening (Han et al., 2015). One of the transferase families, e.g., quercetin-3-O-glucosyl transferases (3-GT), has a role in forming the flavonoid quercetin-3-rutinoside or rutin during tomato fruit ripening (Capanoglu et al., 2012). One important enzyme family of oxidoreductase, or quinone oxidoreductase, is ripening-induced and catalyzes the formation of 4-hydroxy2,5-dimethyl-3(2H)-furanone (HDMF), the key flavor compound in strawberry (Klein et al., 2007). Family of this enzyme has previously been isolated from kiwi, raspberry and tomato as natural products (Schwab, 2013). 
Kyoto Encyclopedia of Genes and Genomes (KEGG) has recently moved the exopolygalacturonase enzyme to the "pentose and glucuronate interconversions" pathway, while the latter two enzymes were moved to "amino sugar and nucleotide sugar metabolism" pathway. The correlation coefficients (R2) ranged in value between 0.9900 and 1.0000, and PCR amplification efficiencies over 94\% were obtained from the standard curves generated using a 10-fold serial dilution of cDNA.

KEGG annotation assigned 846 transcripts to 89 KEGG pathways. The pathway most strongly represented was "starch and sucrose metabolism" (seven activated enzymes). Tomato fruit is very powerful sink for carbohydrate especially in early stages of development. Sucrose is the major photoassimilate transported from photosynthetic leaves to developing fruit. Carbohydrates comprise the most abundant and widely distributed food components derived from plants. Their contents vary greatly in tomato fruit during development and ripening. Selection in this fruit was oriented towards sweetness as sugar, mainly sucrose, glucose and fructose, level ( $\sim 60 \%$ of the total fruit dry weight) is the indicator of ketchup's quality (O'donoghue et al., 1994). Invertase enzyme cleaves sucrose, which may regulate the rate of carbon import to developing tomato fruit (Fei Wang, 1993). It was demonstrated that changes in sugar composition contribute to alterations in fruit size. Mach (Fridman et al., 2004) discussed the role of apoplastic invertase, which contributes to starch accumulation in pericarp and columella tissues, in determining the soluble solids content of mature fruit (Dinar and Stevens, 1981; Schaffer and Petreikov, 1997).

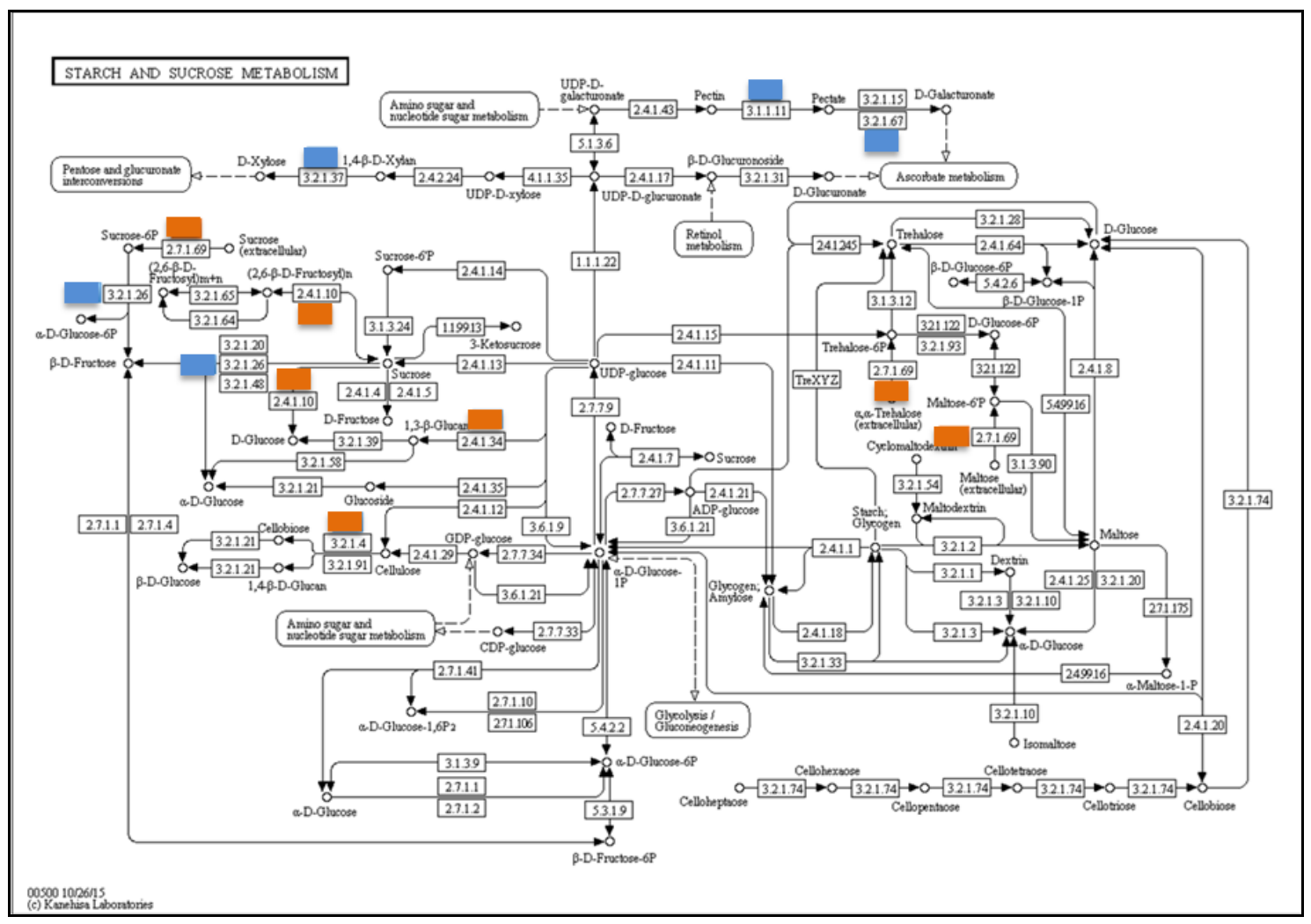

Figure 7. Enzymes in the starch and sucrose metabolic pathway during early stages of tomato fruit development at 12 versus 0 DAP. Upregulated (or activated) (blue box), downregulated (or repressed) (orange box). (Source: https://www.genome.jp/kegg-bin/show_pathway?ko00500) 

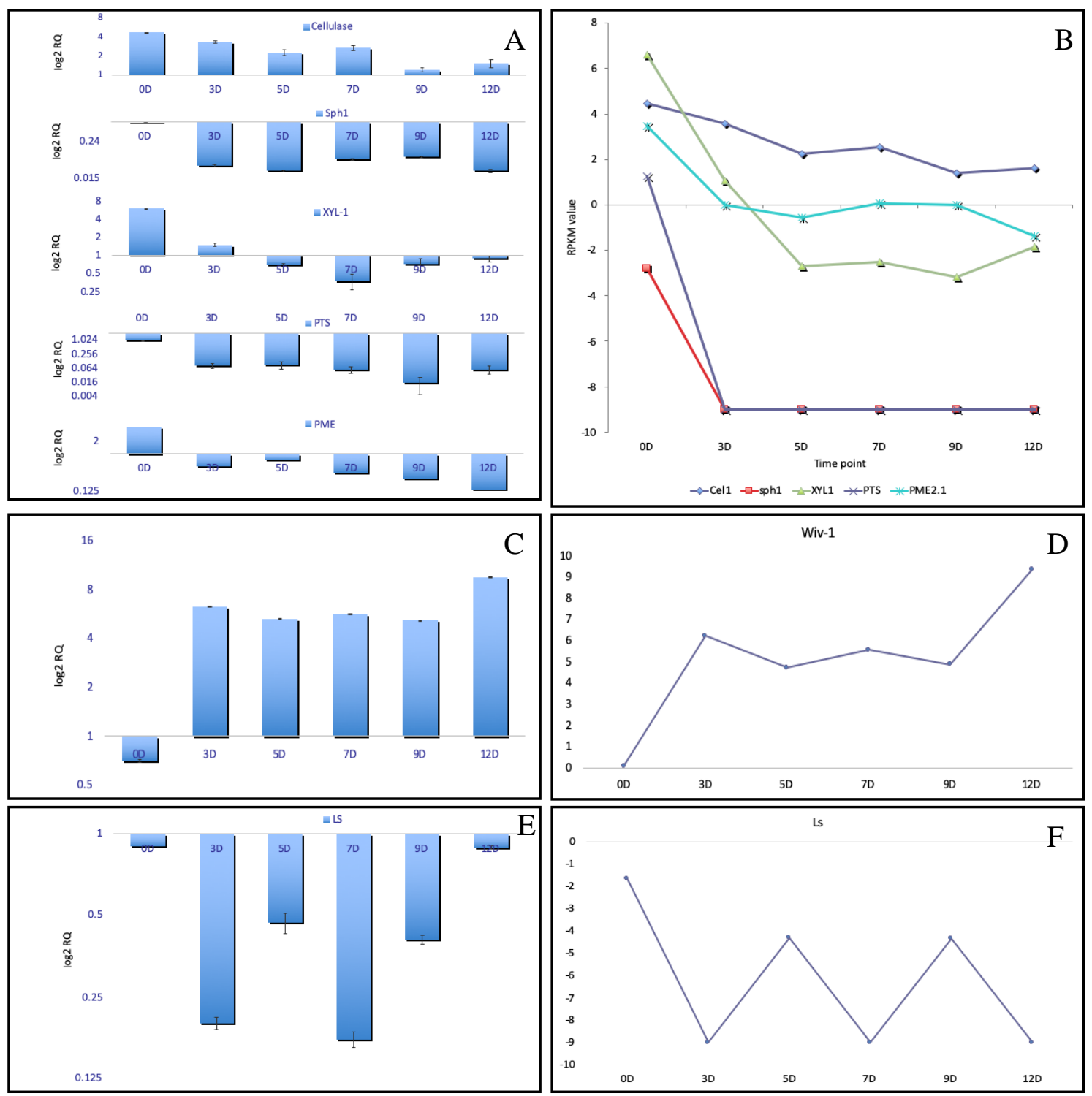

Figure 8. Gene expression verification of PME2.1, WIV-1(sacA), PTS (scrA), LS (sacB), Cellulose (phd) and sphl(cre or Glucan synthase) genes using qPCR. A and B representing the down regulation pattern on both real time PCR results and RNAseq data respectively, $C$ and $D$ representing the up-regulation pattern of WIV-1(sacA), E and $F$ representing fluctuated down regulation of $L S$ ( $\operatorname{sac} B)$ gene expression

Invertase and sucrose synthase, along with the other regulated enzymes of the starch and sucrose metabolism in the present study seem to play a major role during early development of tomato fruits. It is well-known that sucrose, fructose and glucose are stored in the cell vacuole (Mach, 2014).

Figure 8 indicates the overproduction of several soluble sugars, e.g., sucrose, $\beta$-Dfructose, $\alpha$-D-glucose, trehalose, maltose and D-xylose. This conclusion was reached as enzymes upstream these sugars were activated, enzymes downstream them were suppressed or both. Sucrose accumulated due to the suppression of levansucrase (encoded by $s a c B$ ) and phosphotransferase (encoded by $\operatorname{scr} A$ ), while $\beta$-D-fructose and $\alpha$-D-glucose accumulated due to activation of invertase (encoded by $s a c A$ ). High 
activity of the enzyme also results in the synthesis of D-glucose and D-galactose in the "galactose metabolism" pathway (data provided upon request). Trehalose and maltose also accumulated due to suppression of phosphotransferase. In addition, the high activity of the enzyme pectin methyl esterase (PME) can result in approaching "Ascorbate metabolism". Two other enzymes, glucan synthase and cellulase (or endo1,4 $\beta$-D-glucanase), were suppressed. This action results in the accumulation of UDPglucose and cellulose, respectively (Fig. 1).

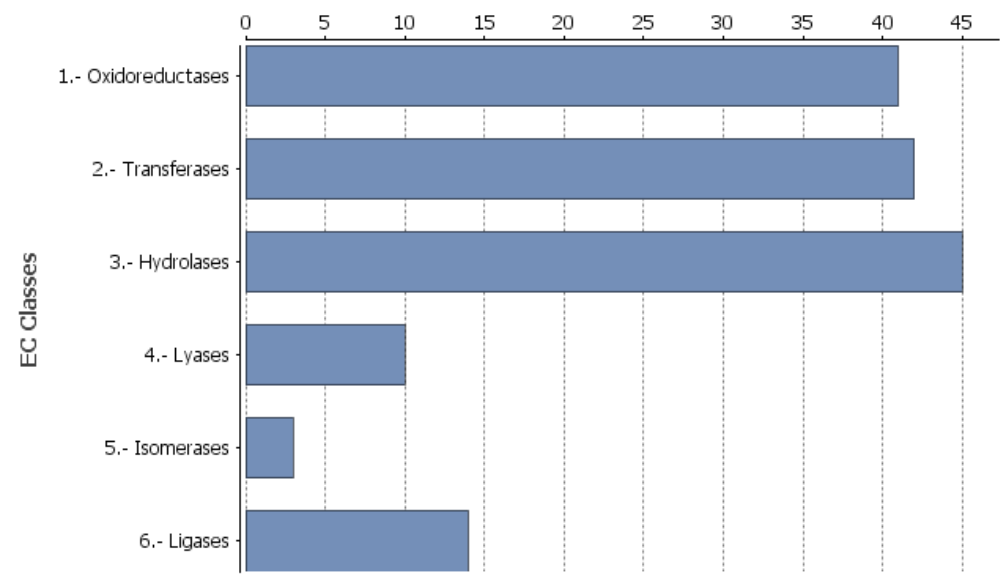

Figure 9. Enzyme code distributions. All transcripts were aligned to the GO database to predict possible enzymatic functions. the total putative proteins were functionally classified into 6 groups

Invertase is encoded by the invertase 5 (or lin5) gene, accumulates in the cell wall and is known to act exclusively in flowers and young fruit (Fridman and Zamir, 2003). The enzyme is important in controlling total soluble solid content in these two organs. Overexpression of the enzyme resulted in increased partitioning of photosynthates to the fruit, hence, enhanced fruit yield (Baxter et al., 2005; Fridman et al., 2004; Schauer et al., 2006).

Zanor et al. (Zanor et al., 2009) reported that knockdown of the gene resulted in decreased levels of glucose and fructose in the fruit. The knockdowns were also characterized by the reduction in fruit size, seed number per plant and levels of phytohormones (LeClere et al., 2008).

Cellulase activity has previously been identified to contribute to cell wall disassembly and fruit softening (Brummell and Harpster, 2001). In avocado, cellulose was also found to contribute to the fruit ripening process (O'donoghue et al., 1994; Pesis et al., 1978). It is likely that this enzyme is suppressed at early stages of fruit development to allow complete maturity before the fruit commits to softening, while high cellulase activity in the abscission zone results in organ abscission in tomato (Brummell et al., 1999; Lashbrook et al., 1994, 1998; Sexton et al., 1980).

$\beta$-d-xylosidase was previously reported to have a role in the hydrolysis of arabinoxylans, xylans, and xyloglucans (Takizawa et al., 2014). The gene was shown to be involved in pear and tomato fruit development and ripening (Itai et al., 1999; Sozzi et al., 2002). The enzyme was also potentially involved in the softening process in strawberry (Fragaria ananassa) fruit (Bustamante et al., 2006). Frenkel et al. (1998) indicated that the production of methanol as well as ethanol in mature tomato fruit is 
positively regulated by activation of pectin methylesterase (PME). The explanation of these results is based on the fact that suppression of the ethanol ADH-catalyzed production is due to methanol accumulation during tomato fruit ripening (Brummell and Harpster, 2001). Earlier reports indicated that mRNA/protein stability and delayed protein synthesis influence the level of PME activity during the development of tomato fruit (Harriman et al., 1991).

\section{Conclusion}

The computational analysis of transcriptomes and heat map showed close relationships among transcripts regulated at 3 and 5 DAP and at 7 and 9 DAP. Transcripts regulated at 12 DAP were more distant compared to the latter four stages. Annotated transcripts and KEGG analysis showed that starch and sucrose pathway experienced the most dynamic change resulting in the synthesis of several soluble sugars and activation of some enzymes. In general, the results provide better insights on the mechanisms underlying early flower and fruit development in tomatoes, which will be valuable for genetic enhancement of tomato fruits in the future.

The authors recommended for further studies to complete all stages involved on fruit repining and development to get complete picture about tomato fruit development.

Acknowledgements. This project was funded by the Deanship of Scientific Research (DSR), King Abdulaziz University, Jeddah, under Grant no. (19-3-1432/HiCi). The authors, therefore, acknowledge with thanks DSR technical and financial support.

\section{REFERENCES}

[1] Bahieldin, A., Atef, A., Sabir, J. S., Gadalla, N. O., Edris, S., Alzohairy, A. M., Radhwan, N. A., Baeshen, M. N., Ramadan, A. M., Eissa, H. F. (2015a): RNA-Seq analysis of the wild barley (H. spontaneum) leaf transcriptome under salt stress. - Comptes Rendus Biologies 338(5): 285-297.

[2] Bahieldin, A., Atef, A., Shokry, A. M., Al-Karim, S., Al Attas, S. G., Gadallah, N. O., Edris, S., Al-Kordy, M. A., Omer, A. M. S., Sabir, J. S. (2015b): Structural identification of putative USPs in Catharanthus roseus. - Comptes Rendus Biologies 338(10): 643-649.

[3] Baxter, C. J., Carrari, F., Bauke, A., Overy, S., Hill, S. A., Quick, P. W., Fernie, A. R., Sweetlove, L. J. (2005): Fruit carbohydrate metabolism in an introgression line of tomato with increased fruit soluble solids. - Plant and Cell Physiology 46(3): 425-437.

[4] Bergervoet, J. H., Berhoeven, H. A., Gilissen, L. J., Bino, R. J. (1996): High amounts of nuclear DNA in tomato (Lycopersicon esculentum Mill.) pericarp. - Plant Science 116(2): 141-145.

[5] Bertin, N. (2005): Analysis of the tomato fruit growth response to temperature and plant fruit load in relation to cell division, cell expansion and DNA endoreduplication. Annals of Botany 95(3): 439-447.

[6] Bonierbale, M. W., Plaisted, R. L., Tanksley, S. D. (1988): RFLP maps based on a common set of clones reveal modes of chromosomal evolution in potato and tomato. Genetics 120(4): 1095-1103.

[7] Brummell, D. A., Harpster, M. H. (2001): Cell wall metabolism in fruit softening and quality and its manipulation in transgenic plants. - Plant Molecular Biology 47(1-2): 311340 . 
[8] Brummell, D. A., Hall, B. D., Bennett, A. B. (1999): Antisense suppression of tomato endo-1, 4- $\beta$-glucanase Cel2 mRNA accumulation increases the force required to break fruit abscission zones but does not affect fruit softening. - Plant Molecular Biology 40(4): 615-622.

[9] Bustamante, C. A., Rosli, H. G., Añón, M. C., Civello, P. M., Martínez, G. A. (2006): $\beta$ Xylosidase in strawberry fruit: isolation of a full-length gene and analysis of its expression and enzymatic activity in cultivars with contrasting firmness. - Plant Science 171(4): 497-504.

[10] Capanoglu, E., Beekwilder, J., Matros, A., Boyacioglu, D., Hall, R. D., Mock, H. P. (2012): Correlation of rutin accumulation with 3-o-glucosyl transferase and phenylalanine ammonia-lyase activities during the ripening of tomato fruit. - Plant Foods for Human Nutrition 67(4): 371-376.

[11] Dinar, M., Stevens, M. (1981): The relationship between starch accumulation and soluble solids content of tomato fruits. - Journal American Society for Horticultural Science 106: 415-418.

[12] Fei Wang, A. S., Brenner, M., Smith, A. (1993): Sucrose synthase, starch accumulation, and tomato fruit sink strength. - Plant Physiol. 101: 321-327.

[13] Frenkel, C., Peters, J. S., Tieman, D. M., Tiznado, M. E., Handa, A. K. (1998): Pectin methylesterase regulates methanol and ethanol accumulation in ripening tomato (Lycopersicon esculentum) fruit. - Journal of Biological Chemistry 273(8): 4293-4295.

[14] Fridman, E., Zamir, D. (2003): Functional divergence of a syntenic invertase gene family in tomato, potato, and Arabidopsis. - Plant Physiology 131(2): 603-609.

[15] Fridman, E., Carrari, F., Liu, Y.-S., Fernie, A. R., Zamir, D. (2004): Zooming in on a quantitative trait for tomato yield using interspecific introgressions. - Science 305(5691): 1786-1789.

[16] Gapper, N. E., Giovannoni, J. J., Watkins, C. B. (2014): Understanding development and ripening of fruit crops in an 'omics' era. - Horticulture Research 1: 14034.

[17] Gillaspy, G., Ben-David, H., Gruissem, W. (1993): Fruits: a developmental perspective. The Plant Cell 5(10): 1439.

[18] Guigó Serra, R., Câmara Ferreira, F., Consortium, T. G. (2012): The tomato genome sequence provides insights into fleshy fruit evolution. - Nature 485(7400): 635-641.

[19] Gupta, S., Shi, X., Lindquist, I. E., Devitt, N., Mudge, J., Rashotte, A. M. (2013): Transcriptome profiling of cytokinin and auxin regulation in tomato root. - Journal of Experimental Botany 64(2): 695-704.

[20] Han, Y., Zhu, Q., Zhang, Z., Meng, K., Hou, Y., Ban, Q., Suo, J., Rao, J. (2015): Analysis of xyloglucan endotransglycosylase/hydrolase (XTH) genes and diverse roles of isoenzymes during persimmon fruit development and postharvest softening. - PloS One 10(4): e0123668.

[21] Harriman, R. W., Tieman, D. M., Handa, A. K. (1991): Molecular cloning of tomato pectin methylesterase gene and its expression in Rutgers, ripening inhibitor, nonripening, and never ripe tomato fruits. - Plant Physiology 97(1): 80-87.

[22] Itai, A., Kawata, T., Tanabe, K., Tamura, F., Uchiyama, M., Tomomitsu, M., Shiraiwa, N. (1999): Identification of 1-aminocyclopropane-1-carboxylic acid synthase genes controlling the ethylene level of ripening fruit in Japanese pear (Pyrus pyrifolia Nakai). Molecular and General Genetics MGG 261(1): 42-49.

[23] Kanehisa, M., Goto, S. (2000): KEGG: kyoto encyclopedia of genes and genomes. Nucleic Acids Research 28(1): 27-30.

[24] Kimura, S., Sinha, N. (2008): Tomato (Solanum lycopersicum): A Model Fruit-Bearing Crop. - CSH Protoc. DOI: 10.1101/pdb.emo105.

[25] Klein, D., Fink, B., Arold, B., Eisenreich, W., Schwab, W. (2007): Functional characterization of enone oxidoreductases from strawberry and tomato fruit. - Journal of Agricultural and Food Chemistry 55(16): 6705-6711. 
[26] Lashbrook, C. C., Gonzalez-Bosch, C., Bennett, A. B. (1994): Two divergent endo-beta1, 4-glucanase genes exhibit overlapping expression in ripening fruit and abscising flowers. - The Plant Cell 6(10): 1485-1493.

[27] Lashbrook, C. C., Giovannoni, J. J., Hall, B. D., Fischer, R. L., Bennett, A. B. (1998): Transgenic analysis of tomato endo- $\beta-1,4$-glucanase gene function. Role of cell in floral abscission. - The Plant Journal 13(3): 303-310.

[28] LeClere, S., Schmelz, E. A., Chourey, P. S. (2008): Cell wall invertase-deficient miniature1 kernels have altered phytohormone levels. - Phytochemistry 69(3): 692-699.

[29] Mach, J. (2014): Modeling sugar metabolism in tomato fruit. - The Plant Cell 26(8): 3222-3223.

[30] Mintz-Oron, S., Mandel, T., Rogachev, I., Feldberg, L., Lotan, O., Yativ, M., Wang, Z., Jetter, R., Venger, I., Adato, A. (2008): Gene expression and metabolism in tomato fruit surface tissues. - Plant Physiology 147(2): 823-851.

[31] O'donoghue, E., Huber, D. J., Timpa, J., Erdos, G., Brecht, J. (1994): Influence of avocado (Persea americana) $\mathrm{Cx}$-cellulase on the structural features of avocado cellulose. - Planta 194(4): 573-584.

[32] Pesis, E., Fuchs, Y., Zauberman, G. (1978): Cellulase activity and fruit softening in avocado. - Plant Physiology 61(3): 416-419.

[33] Picken, A. (1984): A review of pollination and fruit set in the tomato (Lycopersicon esculentum Mill.). - Journal of Horticultural Science 59(1): 1-13.

[34] Robinson, M. D., McCarthy, D. J., Smyth, G. K. (2010): edgeR: a Bioconductor package for differential expression analysis of digital gene expression data. - Bioinformatics 26(1): 139-140.

[35] Schaffer, A. A., Petreikov, M. (1997): Sucrose-to-starch metabolism in tomato fruit undergoing transient starch accumulation. - Plant Physiology 113(3): 739-746.

[36] Schauer, N., Semel, Y., Roessner, U., Gur, A., Balbo, I., Carrari, F., Pleban, T., PerezMelis, A., Bruedigam, C., Kopka, J. (2006): Comprehensive metabolic profiling and phenotyping of interspecific introgression lines for tomato improvement. - Nature Biotechnology 24(4): 447-454.

[37] Schwab, W. (2013): Natural 4-hydroxy-2,5-dimethyl-3(2H)-furanone (Furaneol(R)). Molecules 18(6): 6936-51.

[38] Sexton, R., Durbin, M. L., Lewis, L. N., Thomson, W. W. (1980): Use of cellulase antibodies to study leaf abscission. - Nature 283: 873-874.

[39] Silva, G. F. F. e., Silva, E. M., da Silva Azevedo, M., Guivin, M. A. C., Ramiro, D. A., Figueiredo, C. R., Carrer, H., Peres, L. E. P., Nogueira, F. T. S. (2014): microRNA156targeted SPL/SBP box transcription factors regulate tomato ovary and fruit development. - The Plant Journal 78(4): 604-618.

[40] Sozzi, G. O., Fraschina, A. A., Navarro, A. A., Cascone, O., Greve, L. C., Labavitch, J. M. (2002): $\alpha$-L-Arabinofuranosidase activity during development and ripening of normal and ACC synthase antisense tomato fruit. - HortScience 37(3): 564-566.

[41] Takizawa, A., Hyodo, H., Wada, K., Ishii, T., Satoh, S., Iwai, H. (2014): Regulatory specialization of xyloglucan (XG) and glucuronoarabinoxylan (GAX) in pericarp cell walls during fruit ripening in tomato (Solanum lycopersicum). - PloS One 9(2): e89871.

[42] Trapnell, C., Pachter, L., Salzberg, S. L. (2009): TopHat: discovering splice junctions with RNA-Seq. - Bioinformatics 25(9): 1105-1111.

[43] Trapnell, C., Williams, B. A., Pertea, G., Mortazavi, A., Kwan, G., Van Baren, M. J., Salzberg, S. L., Wold, B. J., Pachter, L. (2010): Transcript assembly and quantification by RNA-Seq reveals unannotated transcripts and isoform switching during cell differentiation. - Nature biotechnology 28(5): 511-515.

[44] Xiao, H., Jiang, N., Schaffner, E., Stockinger, E. J., van der Knaap, E. (2008): A retrotransposon-mediated gene duplication underlies morphological variation of tomato fruit. - Science 319(5869): 1527-1530. 
[45] Zanor, M. I., Osorio, S., Nunes-Nesi, A., Carrari, F., Lohse, M., Usadel, B., Kühn, C., Bleiss, W., Giavalisco, P., Willmitzer, L. (2009): RNA interference of LIN5 in tomato confirms its role in controlling Brix content, uncovers the influence of sugars on the levels of fruit hormones, and demonstrates the importance of sucrose cleavage for normal fruit development and fertility. - Plant Physiology 150(3): 1204-1218.

[46] Zhan, Y., Qu, Y., Zhu, L., Shen, C., Feng, X., Yu, C. (2018): Correction: transcriptome analysis of tomato (Solanum lycopersicum L.) shoots reveals a crosstalk between auxin and strigolactone. - PloS One 13(9): e0204873.

[47] Zhang, S., Xu, M., Qiu, Z., Wang, K., Du, Y., Gu, L., Cui, X. (2016): Spatiotemporal transcriptome provides insights into early fruit development of tomato (Solanum lycopersicum). - Sci Rep 6: 23173. 